\title{
Medical nanobot for cell and tissue repair
}

\begin{abstract}
Nano robots are very tiny machines (too negligible to see through naked eye) designed to perform a specific task whose components are at or close to the scale of a nanometer. Medical nanobots are in the range of nanometer scale $\left(10^{-9}\right)$ and the main element used will be carbon nanotubes because of strength and chemical inertness. This paper discloses one of the major application of a nanobot that can be used for human cell, tissue and even organ repair. Since nanobot performs more than one task for repairing a tissue hence there is a need of programming and usage of more than one nanobots. Cell/tissue repair nanobot is more advanced and sophisticated than nanobot that is used in the cancer treatment.
\end{abstract}

Keywords: nanobot, nano robot, medical nanobot, nonoids, nanites, nano machines, nano mites, cell repair, tissue repair
Volume 2 Issue 6 - 2017

\author{
Manu Mitra \\ Department of Alumnus with Electrical Engineering, University \\ of Bridgeport, USA
}

Correspondence: Manu Mitra, Department of Alumnus with Electrical Engineering, University of Bridgeport, USA,

Emailmmitra@my.bridgeport.edu

\section{Introduction}

Nanobot is a nano robot machine and sometimes it is also called as nanite, which is a mechanical/electromechanical device whose dimensions are measured in nanometers and it is equal or smaller than one micrometer, or to manipulate components on the range of 1 to $1000 \mathrm{~nm}$ in size. Nanobots are so small that they actually interact on the same level as bacteria and viruses do, and so they are capable of building with the very particles of our bodies: atoms and molecules. Nanobot can be equipped with all sorts of tools such as camera (to monitor and to furnish more extensive information about the human body), nano laser and nano chemical (to clean infected area). Not only that, nanobots can be controlled internally and externally in order to perform millions of useful tasks. Nanobot could perform a variety of similarly functions, from disposing away dead cells/tissues at a wound and helping in re-growing tissue so that it heals cleanly and quickly without leaving a nasty scar. It can also help with festering wounds, which could be easily cleared up by an efficient medical Nanobot. ${ }^{1,2}$

\section{Working principle of a nanobot}

Figure 1 Once infected/damaged tissue is identified and then multiple programmed medical nanobots are introduced inside the infected patient body and nanobots automatically detect the infected/ damaged tissue, all the nanobots move to the infected tissue and use laser to remove infected/dead cells attached to the infected tissue. Once all the dead cells are removed and infected area is cleaned by the nano chemical then nanobot start repairing the tissue (similar to WBC (White Blood Corpuscles) does inside our body). All the work such as removing the dead cells, cleaning the infected area and repairing the cells may or may not be done by single nanobot hence we need multiple programmed nanobot. The whole process is so fast that patient can be healed as quickly as few hours for minor wounds and major wounds it may take up to few days to many days to recover completely. Author did not include all the drawbacks of using nanobots in the human body but one should not exceed the maximum prescribed amount of nanobots in the human body because increasing nanobots in the human body will also increase the toxicity in the blood and it may adverse the health of the effected patient. Although nanobots can be programmed to maintain the maximum amount in the human body and once maximum amount of nanobot exceeds the prescribed amount then nanobots are ejected out of the body. ${ }^{3}$

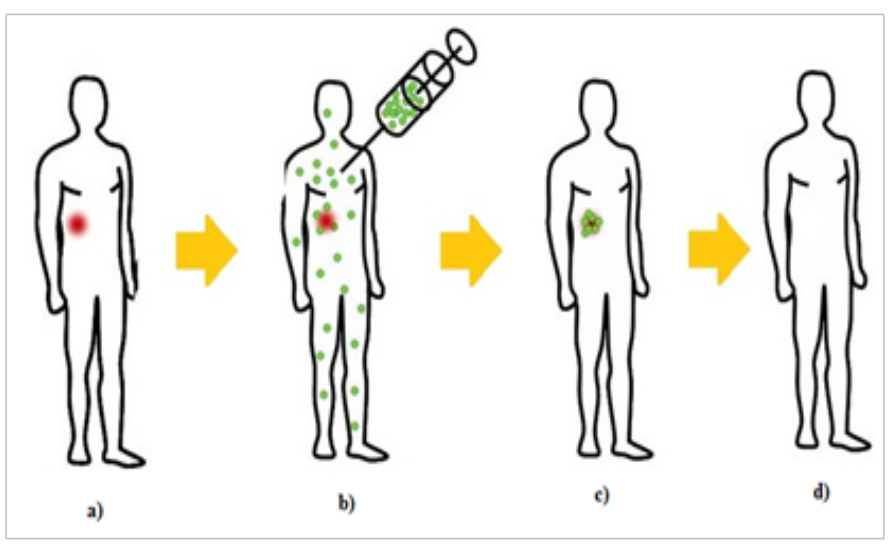

Figure I Depicts that multiple Nanobot heal the damaged tissues. ${ }^{3}$

a. A damaged cell/tissue is detected.

b. Multiple programmed Nanobot inside the human body are introduced.

c. Multiple programmed Nanobot homing the infected/damaged tissue.

d. Damaged tissue has been completely repaired and human body has been recovered.

\section{Design architecture of a basic nanobot}

\section{Major components of nanobot}

Molecular sorting rotor: It is made of carbon nanotube. It is a class of nano-mechanical device capable of selectively binding (or releasing) molecules from/ to solution, and of transporting these bound molecules against significant concentration gradients.

Propeller: It is used for nanobots to drive forward against the blood stream.

Nano camera: Nanobot may include a miniature camera at the size of nanometer.

Lasers: These lasers could burn the harmful material like arterial plaque, blood clots or cancer cells.

Fin: A fin is a surface used for stability and/or to produce lift and thrust or to steer while traveling in water, air, or other fluid media. It is built-in along with the propellers used to propel the device $)^{4}$ Figure 2. 


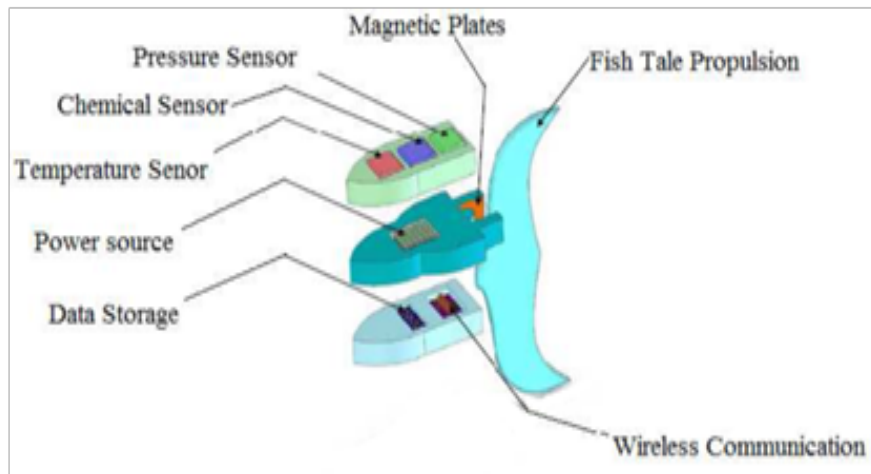

Figure 2 Architecture and basic components of a single medical Nanobot.

\section{Comparison of nanobot for cancer treatment}

Once a cancer cell is encountered, the locks are released, delivering the drug. ${ }^{5}$ Figure 3 shows how nanobot perform for cancer treatment. Similar functions can be sought for cell/tissue repair. Programmed medical Nanobot performs more advanced functions than treatment of cancer.

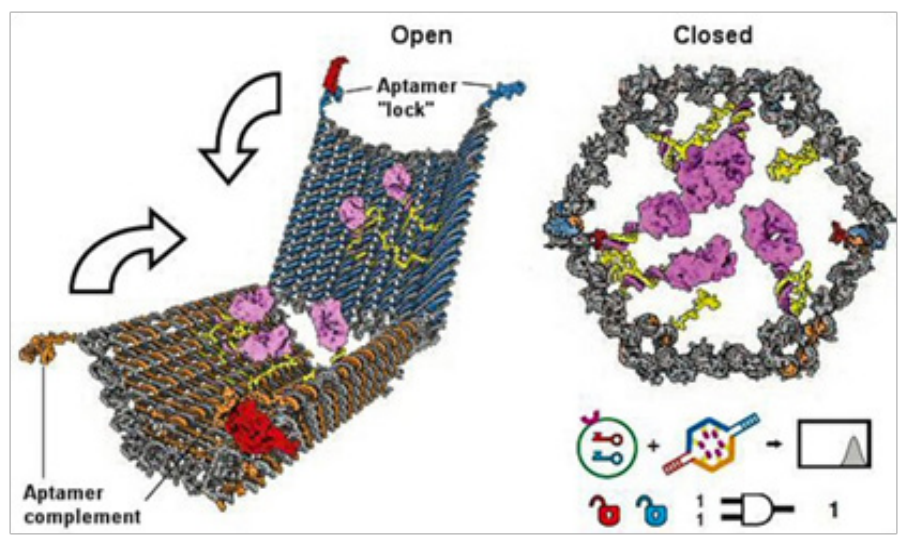

Figure 3 Depicts Nanobot for treatment of cancer.An “open” DNA Nanobot.

\section{Introduction on cell repair of damaged cell}

The size of nanobot can be comparable to the size of viruses or bacteria, their compact parts would allow them to be more complex. It uses powerful nanocomputers and fast sequenators will be needed to guide these nanobot machines. These powerful computers will direct machines to examine, take apart, and rebuild damaged molecular structures. Repair machines will be able to repair whole cells by working structure by structure. Then by working cell by cell and tissue by tissue, whole organs can be repaired. Finally, by working organ by organ, health is restored to the body. Special express DNA-sequenator analyze all DNA and cut off damaged nucleotides, or unwanted genes ${ }^{6}$ Figure $4 \& 5$.

\section{Use of nano laser in the nanobot}

One of the major application of programmed medical nanobot is to repair the damaged cells and tissues. But since one cannot repair a damaged tissue just like that because if the cell is infected, infection would spread to the remaining parts of the body and may cause secondary complication even if it does infect any other cells. Author highly recommends that removal of dead/infected cells is very important and once nanobot removes infected cells including harmful bacteria, virus using nano laser and nanobot has to store infected cells to appropriate place (in the nanobot storage itself) and dispose it appropriately so that infection would not spread to other parts of the body.

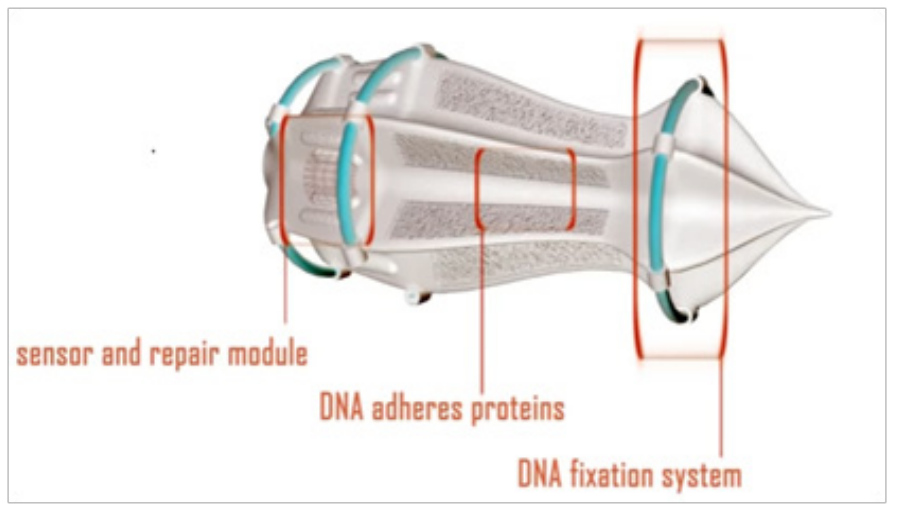

Figure 4 Design Concept of single medical Nanobot.

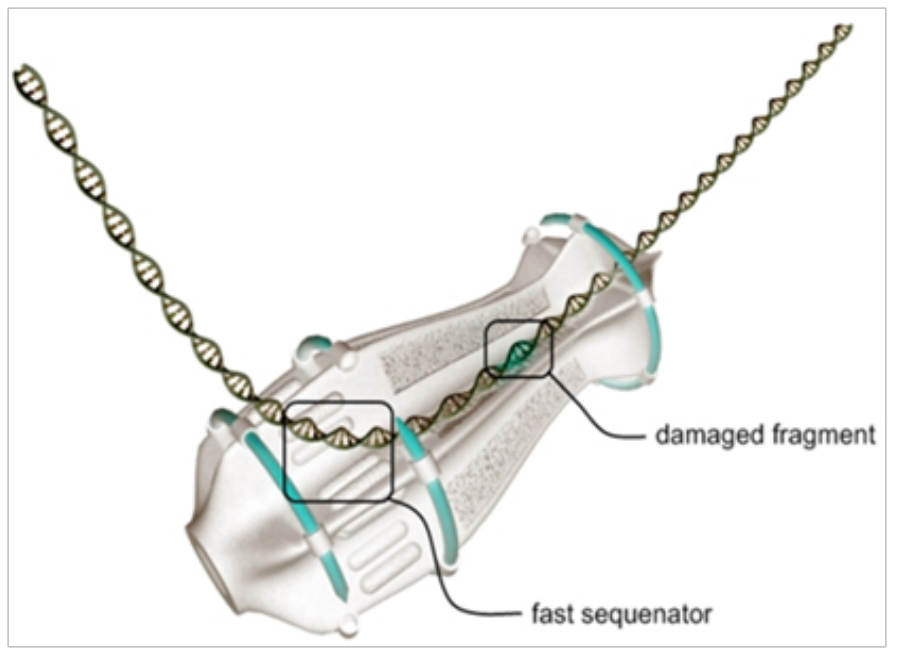

Figure 5 Depicts the Nanobot repairing damaged fragment.

\section{Cleaning the infected area}

Author also highly recommends that after removal of dead and infected cells cleaning is the second step. In this stage nanobot clean the area with nano chemical to avoid any remaining spores does not spread to the other cells.

\section{Repairing the cell/tissue}

After cleaning the area with nano chemical third step is to repair the cells. Nanobots can start repairing the tissue as they are programmed. Here since there are multiple work is involved hence there is a need of more than one nanobot for completion of process successfully.

\section{Power source for the nanobot}

Most of the nanobot require internal and external power sources because of the complexities of its job.

\section{External power source}

Nanobot have to depend on external power source which is already in the human body such as blood flow. With this, nanobot can more easily move throughout the system. But one has to note that it has 
to program these nanobots to move to the designated area. Another power source that nanobots can use is the heats generated from our body that can be recharge the nanobots.

\section{Internal power source}

It is also recommend that there is a need of separate rechargeable battery integrated into the nanobot to perform various functions and can be recharged with not only blood flow, production of heat but also with the blending of chemical reactions in human blood and chemicals in nanobots will lead to the creation of fuel., ${ }^{7,8}$

\section{Programming nanobots}

The Athelas language: Nanobot performs various tasks such as moving compounds between locations in the body, picking compounds (by molecular binding), or exposing (and sometimes releasing) them in affected areas. To perform these tasks, there is a rule-based programming paradigm, in which programs are specified by sets of rules that are continuously considered in parallel, against changing conditions.

Each rule has four clauses. The Initialize clause specifies the set of payloads to be built when it is injected (i.e., before any action is taken). The When and Until clauses are each composed of a set of tests, e.g., $\mathrm{pH}$ level or concentration of a specific chemical in specific location. The When tests must hold in order for the compound to become activated. The Until clause terminate the activity of the compound. The Actions clause contains the actions to be executed when the compound is active, e.g., pick, drop, protect, expose, disable and other actions. In Figure 6, there are two drop actions. One to drop the compound $\$ \mathrm{Z}$ which the compound initially contains, at location @ T. The other drops \$Y at the location @ (A AND B AND C), i.e., a location marked by the presence of all biomarkers A. For instance, the nanobot is capable of dropping a payload in a location marked by at most two markers (e.g., A AND B), and it cannot selectively drop only $\mathrm{Z}$ or expose only $\mathrm{X}$ in a location. Thus in order to have nanobots execute this rule, a mixture of different nanobots (a heterogeneous nanobot) is needed. The role of the compiler is to synthesize this many nanobots, choosing between multiple options if possible to optimize cost, yield, and reliability.

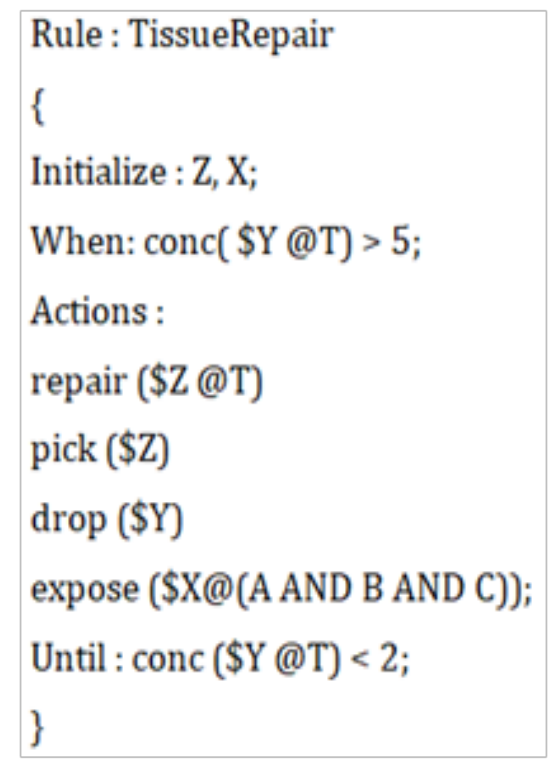

Figure 6 Depicts the rule based programming for tissue repair.
The Bilbo Compiler: The Bilbo compiler takes two inputs: an Athelas program, and a library of generic robot types (with defined ways of parameterizing them, including parameterizable preparation protocols). It then synthesizes a specification for a heterogeneous specialized nanobots, which would carry out the program, once deployed. The output specification for each specialized robot includes a specialized preparation protocol. The compilation process is done in two phases. A front-end phase consists of the lexical and syntax analyzers, generates finite state machines (FSMs) representing the rules. The back-end phase then transforms such FSMs into a final nanobot's specification (recipe). This is done by a graph-rewriting approach, with specific operators for merging, expanding FSM transitions and states, and rejecting incorrect paths. At the end of this process, an AND/OR graph emerges, which represents all possible nanobots that can carry out the program. The compiler uses the AO* algorithm to determine an optimal AND/OR path in the graph, which corresponds to a specific heterogeneous nanobots, made of specialized nanobot archtypes and their preparation protocols..$^{9,10}$

\section{Applications, advantages and disadvantages \\ Applications}

The following are the sample list of applications to start with but there are many other useful tasks that nanobots can perform that are not listed here.

a. Removing Kidney Stones and Liver Stones

$b$. To cure skin diseases

c. Neuron replacement

d. It would prevent heart attack, kill cancer cells etc

$e$. It would augment immune system by finding and killing bacteria and viruses

$f$. It can be used as mouthwash to do all brushing and flossing

g. Smart anti-cancer Therapeutics

h. Parasite removal

$i$. Targeted drug delivery

j. In treatment of Arteriosclerosis

\section{Advantages}

There are many advantages of nanobots and one of the major advantage that author wants to point it out is that patient's whole tissue can repaired in few to some hours rather than in weeks or months and if programmed properly whole damaged organ can be repaired in few days.

Another advantage is that once the tissue is repaired/healed completely, nanobots are not required to stay in the body forever, they can be moved out through programming.

Below are the some of the advantage.

a. Nanobot might function at the atomic and molecular level to build devices, machines or circuits known as molecular manufacturing.

b. Nanobots might also produce copies of themselves to replace worn-out units, a process called self-replication.

c. The microscopic size of nano machines translates into high 
operational speed

d. Individual units require only a tiny amount of energy to operate

e. The major advantage of nanobots is thought to be their durability, in theory, they can remain operational for years, decades or centuries.

\section{Disadvantages}

One of the major disadvantage that author want to make a note is that increasing nanobots in the body will also increase the toxicity in the blood and may adverse the health of the effected patient. It should be noted that the maximum amount of nanobots in the human body should not exceed the prescribed amount.

Below are the some of the disadvantage:

a. Expensive technology

b. Complicate design

c. Initial design cost is very high

d. Hard to program

e. Limited external control mechanisms

f. May affect human health by introducing toxicity in blood

\section{SWOT (Strength Weakness Opportunities Threat) analysis}

Strength: Since it is a new method to treat a patient using nanobots there are not many competitors involved and health care in United States still requires lot of attention.

Weakness: Since it is new way of treatment of patient there are still lot of improvements to be made like how it be better programmed, how many diseases it can be used etc., we also lack in resources right from funding the project to improving the product for future.

Opportunity: In United States Health Care is on the highest priority and there is a lot more to not only improve the Health Care but also need to add more and more technologies for betterment of mankind.

Threats: There are many threats that are required to overcome since it has to be developed, tested, deployed and pre-production testing has to be completed which itself is considerable threat. There will be also interesting to see what would be the government regulations on these new technologies.

\section{Data analysis}

\section{Historical data}

Below is the data taken from Health and Human Services: If we analyze the graphs based on the health coverage in not even in all of the United States. Author points out that not all people in United States are covered with the health care equally and in Author's opinion this technology is independent of any health care coverage and can be used once approved Figure 7.

\section{Current data}

There was survey taken on Nanobot technology. Author expected ten members and actual members were eleven. Below are the graphs that can be interpreted. Based on the interpretation of above graphs and its response it is noted that most of the members would think this technology would be benefit for human mankind. Form for this data can be viewed on the google form (https://goo.gl/forms/ aiyjCuiScZzQBfeq1) Figure 8-12.

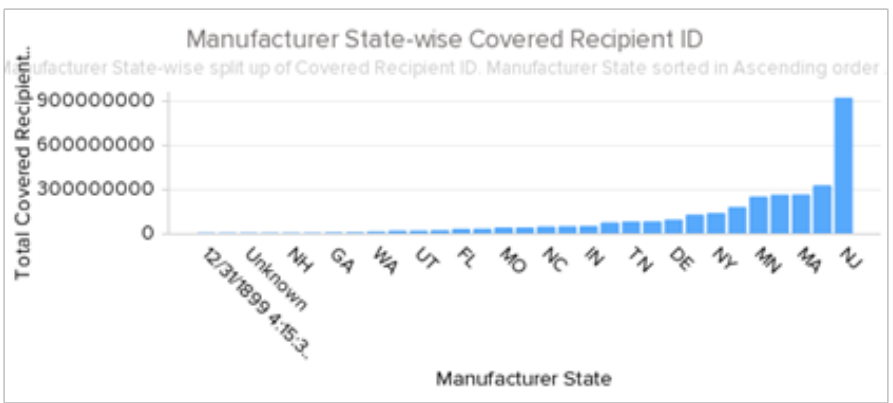

Figure 7 Graph that depicts the health coverage of recipients (Data as of year 2015).

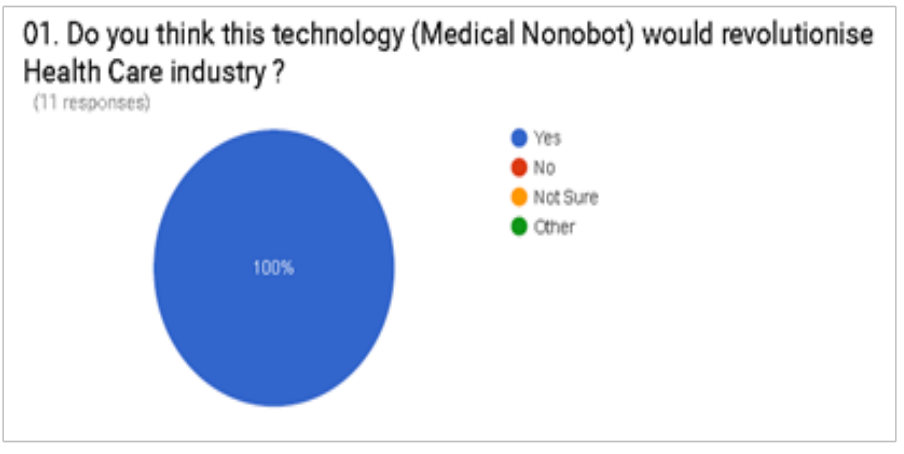

Figure 8 Pie Graph that depicts members accept it will revolutionize Health Care industry.

02. What is the major industry that this technology will this effect? (11 responses)

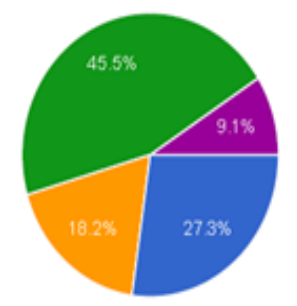

- Medical industry

- Robotic industy

Nanotechnology

- All the Above

- None of the Above

other

Figure 9 Pie Graph that depicts member's response in technology that it effects.

03. If done properly, author believes that many lives can be saved which some of them can't be done antibiotics. Do you agree?

(11 responses:

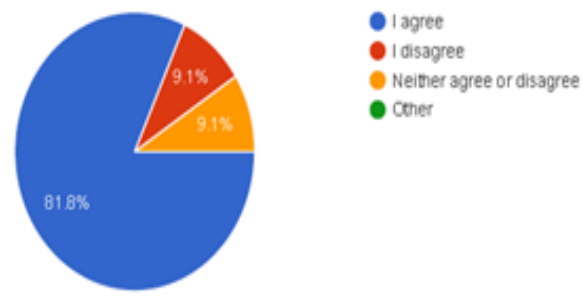

Figure 10 Pie Graph that depicts member's response that this technology can save that some of them can't be done in antibiotics. 


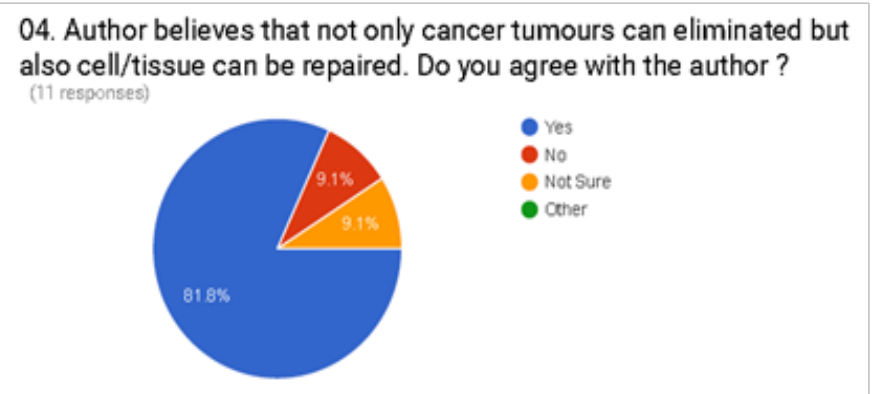

Figure I I Pie Graph that depicts members response that this technology for cell/tissue repairs.

05. On the scale of 1 to 10 how would think that this technology (Medical Nanobot for Cell Tissue Repair) would benefit human mankind? ( 1 being the lowest and 10 being highest)

(11 responses)

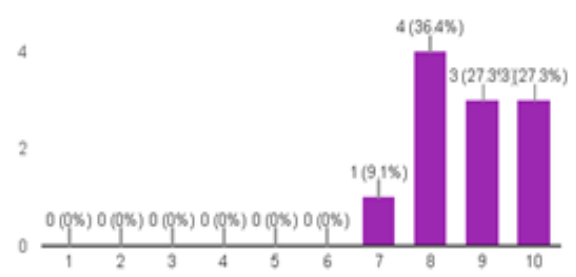

Figure 12 Bar Graph that depicts members' response that this technology would benefit human mankind.

\section{Conclusion}

What is claimed in this are

a. A multiple programmed medical nanobot which is constructed from carbon nanotube and performs many to multiple functions such as removing infected/dead cells attached to the live tissue (using nano laser), cleaning the infected cells (with nano chemical) and repairing the damaged tissue.

b. Nanobot in the claim 1, wherein said performs single to many functions in repairing cell, tissue and organ. c. Nanobot in the claim 1, wherein said multiple programmed medical nanobot requires more than one nanobot to perform all the functions successfully.

d. Nanobot in the claim 1, wherein performs many functions based on the programming of the nanobot.

e. Nanobots in the claim 2, wherein said single to many functions also includes eliminating harmful bacteria, virus spreading other parts of the body.

\section{Acknowledgement}

Author would like to thank Prof. Navarun Gupta, Prof. Hassan Bajwa and Prof. Linfeng Zhang for their academic support. Author also thanks anonymous reviewers for their comments.

\section{Conflict of interest}

Author declares that there is none of the conflicts.

\section{References}

1. Introduction to Nanotechnology. Nano Gloss.

2. Shalini. Nanobots as therapeutic Devices. Slide Share. 2014.

3. Design Architecture of Nanobot. Smart DNA mounts.

4. Rigivendra kumar vardhan. Nano Robotics. Slide Share. 2015.

5. Manjunath Apoorva, Vijay Kishore. The Promising Future in Medicine: Nan robots. Science and education. 2014;2(2):42-47.

6. Jurica Dujmovic. Nanobot are waiting in the wings to cure cancer and clean up ocean pollution. 2016.

7. Yog. Power Sources of nanorobots. Robotics Bible. 2012.

8. Thomas E, Mallouk, Ayusman Sen. Powering Nanorobots. Nano Technology. 2009. p. 1-6.

9. Inbal Wiesel, Noa Agmon, Gal A Kaminka. A Compiler for Programming Molecular Robots. Bar Ilan University. Israel. p. 1-2.

10. Inbal Wiesel-Kapah, Gal A Kaminka, Guy Hachmon, et al. RuleBased Programming of Molecular. Proceedings of the Twenty-Fifth International Joint Conference on Artificial Intelligence. 2016. p. 1-8. 